American Journal of Applied Sciences 6 (4): 752-756, 2009

ISSN 1546-9239

(C) 2009 Science Publications

\title{
Cementation Analysis by Eddy Current Method
}

\author{
M. Zergoug, H. Oubouchou and N. Amimeur \\ Division des Procédés Electriques et Magnétiques \\ Centre de Soudage et de Contrôle, Route de Dely Ibrahim, B.P: 64, Chéraga, Alger
}

\begin{abstract}
Increase the hardness, the endurance and the life time of material can be realised by surface treatments and remetallings, the electromagnetic properties of steels depend on their composition, their microstructures and constraints applied. We can use the electric and magnetic parameters to evaluate their microstructure. The object of this work is the characterization of cementation by the nondestructive methods and the determination of physicochemical parameters. Samples of low carbon tenor steel were cemented with different alternatives. We studied these samples by eddy current method. The treatment of these results will be able to inform us about the cementation parameters. This work allow to determine the surface layers by NDT methods.
\end{abstract}

Key words: NDT, cementation, carbon, eddy current, Micro hardness

\section{INTRODUCTION}

The use properties of the metallic materials are slightly linked to their micro structural status. The characterisation of this microstructure a significant assets to the industrial, who wants to check the quality of the elaborated material. The reference method chosen to compare our results is the profile of microhardness. Following concreting of the carbon concentration drops asymptotically from the surface by going through the core. It is the same for the hardness and as its profile of hardness which conditions the performance of the operating parts, it is then naturally to the hardness required to check the concreting. The determination of the micro-hardness by the classical method destructive presents a disadvantage other the fact that it is destructive, its shown to be slow and constraint, it needs the preparation of samples by cutting, polishing and possibly layering. Our work consists of characterising the concreted steels by non destructive electromagnetic methods, this bibliographical section touches on the materials used steel on one hand and on the non destructive characterisation methods. In the same time to rising the cooling speed, the ferrite and pearlite components which are formed at lower temperatures and become thinner. The sooner the cooling speeds are higher, we get a carbon ferrite aggregate of which the morphology is so different from that of the pearlite, that we call bainite. When steel is cooled more rapidly to a speed higher to certain critic speed said «tempering critical speed», the austenite transforms without any intervention of diffusion in centred quadratic phase in which the carbon remains in solution and this one is called martensite.

Aim: The aim of this double treatment is getting, to the surface of steel, of a layer to 0.3 at $2 \mathrm{~mm}$ thick to a high content in carbon. The content generally comprised between $(0.6$ and $0.9 \%)$. The layer in question presents a structure essentially martensitic of bigger hardness able to reach 700 to $900 \mathrm{HV}$ in surface and high compression residual, it is capable to stand severe mechanical solicitations, lies on a core having a hardness clearly less higher inferior or equal to nearly $400 \mathrm{HV}$ and presents good tenacity characteristics.

The treated steels: They are the steels that are part of the steels used for thermal treatments, they present a fine grain and low content in carbon and they are classified in six families. The treatments implemented for the hardening of the surface layers also cause the formation, in surface, of the compression residual constraints of which the level and distribution play a leading role in the performance in service of the parts, particularly in the phenomena of tiredness and contact. The X-rays constitute the option method for the study of the residual constraints. The existence of relations between the metallographic structure nature of phase, dimension of the ranges of the various phases in the multiphase structures and the values taken by the physical parameter having a influence on the eddy

Corresponding Author: M. Zergoug, Division des Procédés Electriques et Magnétiques, Centre de Soudage et de Contrôle, Route de Dely Ibrahim, B.P:64, Chéraga, Alger Tel: (213)(21) 361854 à 55 Fax: (213)(21) 361850 
currents permits to come up through these values to the corresponding microstructures or, at least, to their local variation, provided that the all the other influence parameters have been controlled.

Eddy current: The method of eddy current is very sensible to the least variation in the properties of the conductor materials. However, not many studies have been carried out concerning the use of this method for the characterisation of these properties. This method is usually used in the field of detection of faults in the metallic materials. The eddy currents can be influenced by the variation of the conductivity which is electric and influenced by various metallurgical factors such as the microstructure, the variation of the temperature, the change in the hardness.

A study including the cemented steel of type $10 \mathrm{NiCr} 6$ concerned the determination of cementation thickness. The results obtained show that the procedures through eddy currents can be correlated in a linear way with the evolution of the character thickness of cementation ${ }^{[1,2]}$. Other studies concerned other parameters such as the structural status and hardness. The Rao works show that the variation of the microstructure in the austenitic stainless steels can affect the magnetic permeability and electrical conductivity of the material. However, he concentrated his studies on the detection of the faults ${ }^{[3]}$ the studies show that the eddy currents can be used to determine the micro structural status of the materials. The eddy currents are affected by the grain size variation, of the microstructure and the hardness. The micro-hardnesses were qualified according to the electrical impedance of the receiver coil. As for the ferromagnetic materials, two parameters have to be taken into consideration: the electrical conductivity and the magnetic permeability. The use of the low frequencies in these materials gives a good resolution thanks to the separation of these two parameters. These studies show a relationship between the micro-hardness and impedance, the impedance increases with the micro-hardness. The two sections, real and imaginary, are influenced by the variation of the impedance due to the thermal treatment of the ferromagnetic materials.

\section{MATERIALS AND METHODS}

Experimental approach: Three differences of steel were purpose of our study, it is about differences marketed under the following descriptions: $10 \mathrm{NiCr} 6$, $16 \mathrm{NiCr} 6$ and 20NiCr6. We selected nickel-chrome steel apt for cementation, available and having chemical compositions brought together outside the content in carbon. This choice is justified by the important use of the later in industry, particularly in the manufacture of sprockets. We have three cylinder bars having respectively the following diameters, $20 \mathrm{~mm}$ for the difference $20 \mathrm{NiCr} 6$, 60 for the $10 \mathrm{NiCr} 6$ and $100 \mathrm{~mm}$ for the $16 \mathrm{NiCr}$. We prepared six samples by difference by cutting the washers of $10 \mathrm{~mm}$ thick. Five samples by difference were subject to cementation treatment of the 6th serving as reference. With different maintenance times, to a temperature comprised between 900 and $1000^{\circ} \mathrm{C}\left(\mathrm{Ac}_{3}\right)$ and in a carburetting, mixture of gas and oil, we obtained five different thicknesses of layers cemented by difference. The cementation was carried in three phase.

\section{RESULTS AND DISCUSSION}

Micro-hardness filiations: (Realised on basis of the NFA 04-202 standard): This test permits us to determine the evolution of the hardness in depth from the surface. Micro-hardness measurements are taken from the periphery to the centre with a pace of $50 \mu \mathrm{m}$. the distance between both tests is $100 \mu \mathrm{m}$. According to these results, we deduct the depth of the cementation to each sample as well as surface hardness, hardness to $100 \mu \mathrm{m}$ of the cemented surface, and the hardness to the core, heart. As we show in the Fig. 1 the 10NiCr6, $16 \mathrm{NiCr} 6$ and $20 \mathrm{NiCr} 6$ presenting both values of surface micro hardness brought together whatever is the maintenance time. As for the differences in the cementing medium acting then as a source of surface carbon fixing the content in surface of this element in phase $\gamma$. Thus, the cementing medium acted as a source of surface carbon, the content in surface of this element in phase $\gamma$ is in increase with time. The variation of the surface hardness on basis of the maintenance in the cementing medium confirms this hypothesis. The results obtained in this phase permit us to interpret the results obtained by the non destructive methods.

Impedance diagrams: The results of measurements are presented in the normalised impedance plan for the frequencies going from $100 \mathrm{~Hz}-100 \mathrm{kHz}$. This wide scale of frequency, permit us to examine our samples to various depths. These representations on Fig. 1 show that a change in cementation depth, or absence of the latter, displaces the point in the different itineraries, of a given frequency. The diagrams of impedance obtained permit us, however, to confirm the stability of our sampler. The Fig. 2 related to three differences studied present the variation $\Delta \mathrm{Z}$ impedance of cemented sample-the non cemented impedance samples on basis of the thickness of the cementation. Thickness obtained by the micro hardness measurements. The variation de 

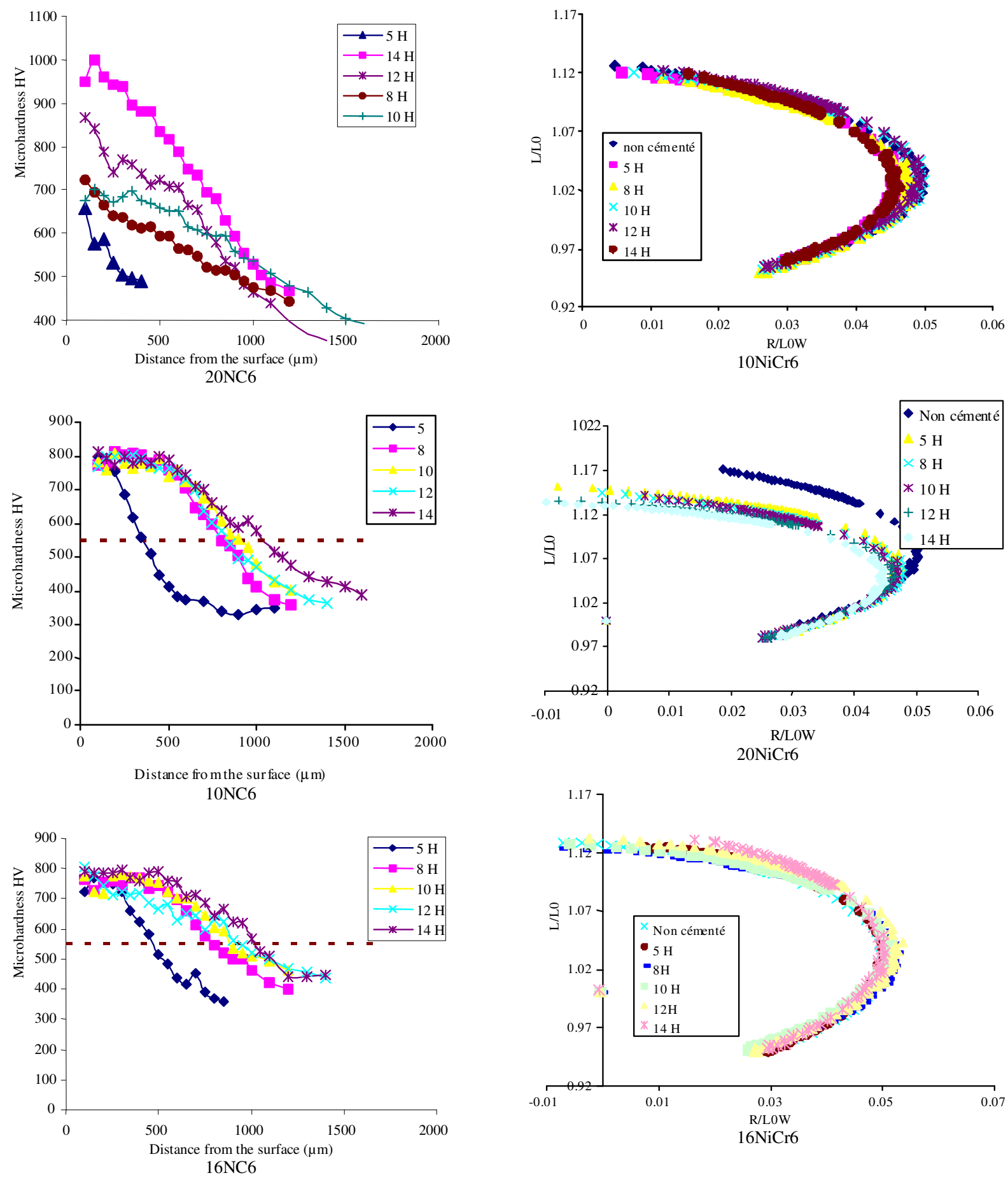

Fig. 1: Filiations of cementation steel according to different time

$\Delta \mathrm{Z}$ depends on the frequency. We notice that for the three differences that the evolution of impedance on basis of the thickness of cementation differs from

Fig. 2: Impedance Diagram of cementation steel according to different time cementation

frequency to another. This confirms that the treatment that the treatments applied amended the microstructure of the samples. The use of a low frequency permits a deepest penetration of eddy currents. The cementation, 
Am. J. of Appl. Sci., 6 (4): 752-756, 2009
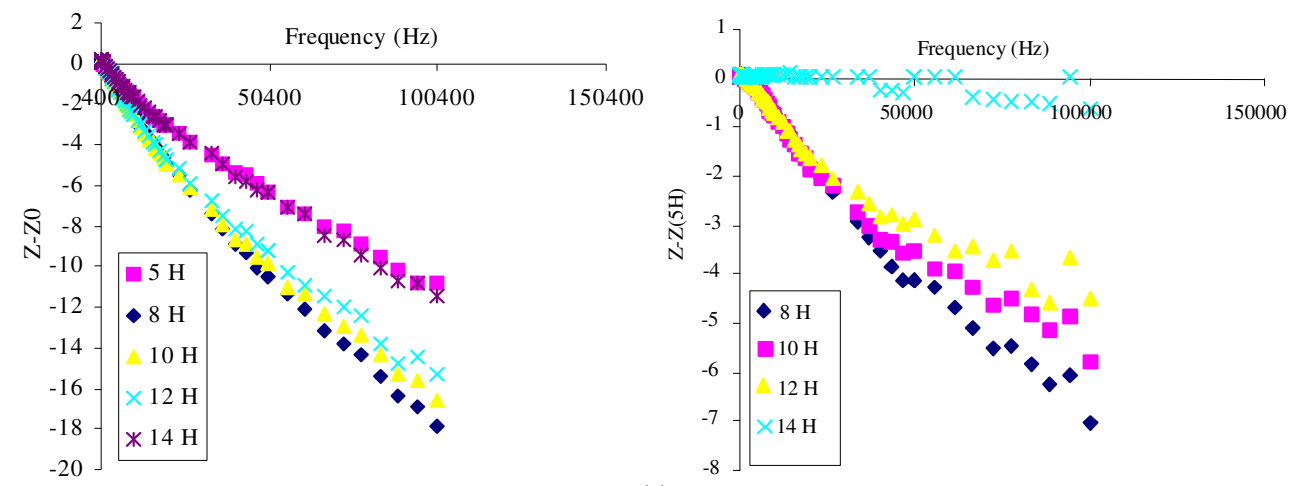

(a)
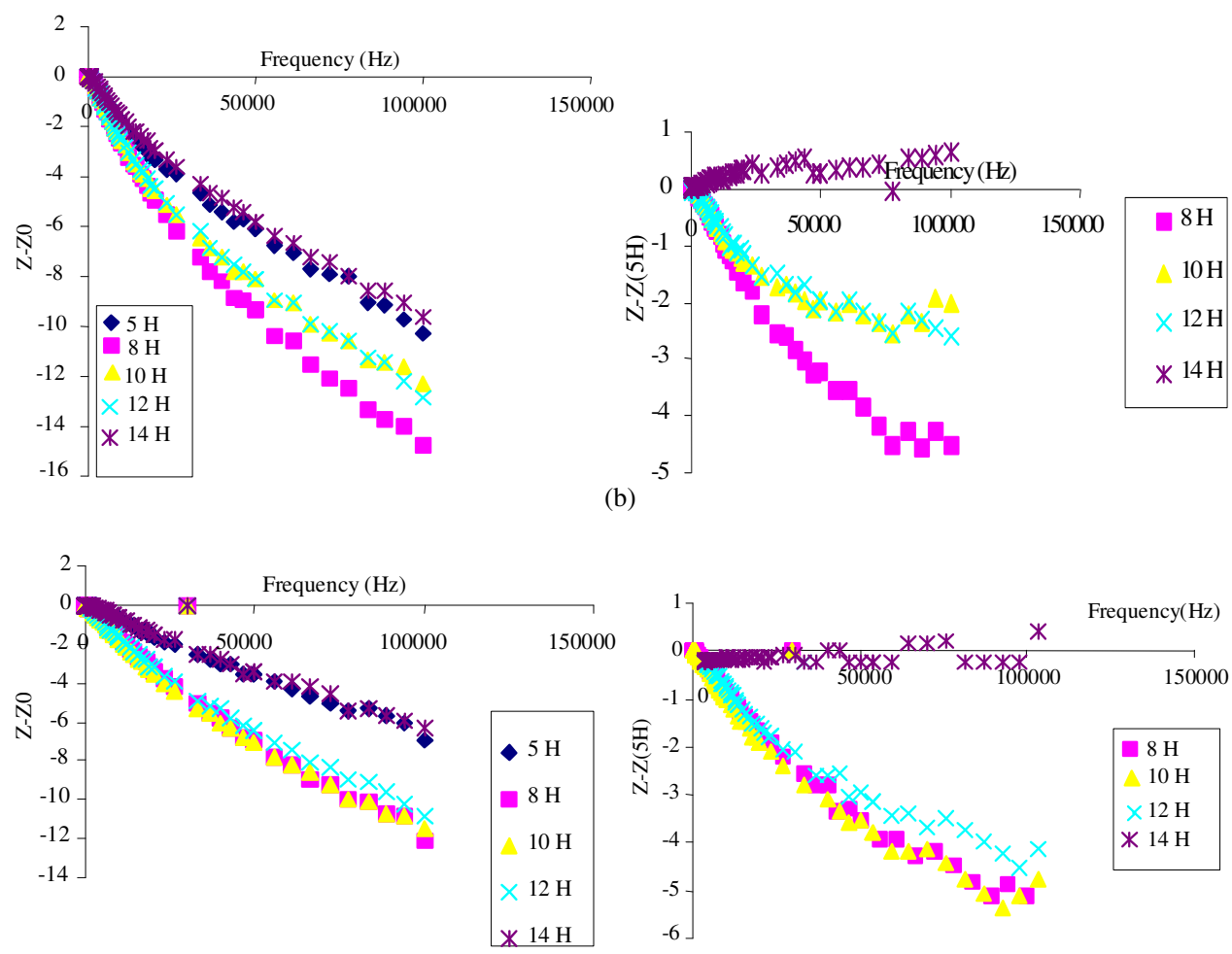

(c)

Fig. 3: Variation $\mathrm{Z}-\mathrm{Z}_{0}$, $\mathrm{Z}-\mathrm{Z}(5 \mathrm{H})$ variations to the frequency according to time cementation. (a): 10NiCr6, (b): $16 \mathrm{NiCr} 6,(\mathrm{c}): 10 \mathrm{NiCr} 6$

enriched in carbons the surface layers which leads to a local drop of the values of $\sigma$ and $\mu$ and by way of consequence should be sent to drop of the values of the impedance $\mathrm{Z}\left(\mathrm{Z}-\mathrm{Z}_{0}<0\right)$. This is checked, according to the Fig. 3, for the higher frequencies. The effect of skin, necessary characteristics of the eddy currents, plays an important role in the interpretation of results, this effect tends to concentrate the circulation of currents induced more and more in the surface when the frequency increases. The treatment of the cementation creates a gradient of chemical composition content in carbon which involves itself after tempering a gradient residual constraint concentration of compression residual constraints in surface and micro structural components $\mathrm{x}$ variation of martensite rate or its morphology, bainite, residual austenite or pearlite, of the surface till the core. These variations influence the impedance measurements. In order for taking into consideration these micro structural brought to by the tempering in addition to the variation of the content in carbon, we 
present on the Fig. 3, on basis of the frequency, the variation e, la variation de $Z-Z_{0}$ on one side and that of $\mathrm{Z}-\mathrm{Z}_{5 \mathrm{H}}$ on the other side, avec $\mathrm{Z}_{0}$, impedance of the sample which was not subject to any treatment and $\mathrm{Z}_{5 \mathrm{H}}$ : impedance of sample having the lowest cementation thickness. In fact, the curves representing $\mathrm{Z}-\mathrm{Z}_{0}$ do not follow a particular order but have the same evolution than the results obtained by the curves giving the profile representation of the micro hardness on basis of the depth in surface. However, those representing Z$\mathrm{Z}_{5 \mathrm{H}}$ are more and more shifted to top when cementation time increases and this is valid for the three differences studied. We notice as well that $\mathrm{Z}-\mathrm{Z}_{5 \mathrm{H}}$ variations are more and more important as the frequency increases. These results lead us to plot the $\mathrm{Z}-\mathrm{Z}_{5 \mathrm{H}}$ variations related to the some frequencies, on basis of maintenance time and also on basis of the thickness of cementation, considering that this thickness which conditions in a big part the performance in service of a cemented part. We notice that the linear of the results obtained. The right of the least squares obtained presents a low error.

\section{CONCLUSION}

The check by the electromagnetic methods takes an important place in the solving of the complicated problems of characterisation of materials. These methods are very demanding in the industry thanks to the economic benefits and other benefits in time that they bring. We studied the in this work the possibility of characterising the cemented steels by using the eddy currents. This method presents, thanks to its simplicity, a solution for the characterisation of this kind of treatment, considering complexity, the content and destructibility of the classical means used for this purpose that is the determination of the micro hardness profile. The results obtained with the eddy currents show that this method adapts itself to this kind of treatment.
The eddy currents offer the possibility of determining the thickness of cementation by plotting calibration curves connecting $\Delta \mathrm{Z}$ impedance variation according to a sample having been subject to same tempering. To this thickness, they also permitted to determine the scale of the adequate frequency. The best results are obtained with the highest frequency. We found out a link between the micro hardness profile and the impedance measurement. The advantage of the eddy currents is that it permits by means of the frequency variation to come back towards more precise micro hardness profiles, Inferior to 50 microns. This result is interesting from the practical side, but does not permit a full characterisation of the cemented layer, it does not permit to estimate the chemical and micro structural value of the cemented layer.

\section{REFERENCES}

1. Zergoug M., S. Mebrek and M.R. Chennou, 1997. Building and testing of probes for the non destructive characterization by eddy currents of cementation coating steels. Ann. Chim. Sci. Mater., 22: $169-179$.

2. Chenno, M., 1994. Caractérisation des couches de cémentation par les courants de Foucault. Mémoire de Postgraduation.

3. Baldev Raj, V. Moorthy, T. Jayakumar and K. Bhanu Sankara Rao, 2003. Assessment of microstructures and mechanical behaviour of metallic materials through non destructive characterisation. Int. Mater. Rev., 48. 